\title{
CINÉTICA E MODELAGEM DA SECAGEM DA UVA CRIMSON EM SECADOR CONVECTIVO
}

\author{
A.R.S.TELES ${ }^{*}$, A.M.CONCEIÇÃO ${ }^{1}$,G.S.SILVA ${ }^{1}$, S.P.S.SANTOS ${ }^{1}$, R.G.SANTOS ${ }^{2}$, G.F.SILVA ${ }^{3}$. \\ ${ }^{1}$ Universidade Federal de Sergipe, Departamento de Tecnologia de Alimentos \\ ${ }^{2}$ Universidade Federal de Sergipe, Engenharia Química \\ ${ }^{3}$ Universidade Federal de Sergipe, Laboratório de Tecnologias Alternativas \\ *email: alan122006@hotmail.com
}

\begin{abstract}
RESUMO
A secagem consiste do processo remoção de água e este processo pode ser descrito por uma série de modelos semiteóricos. Este trabalho teve como objetivo obter a curva de secagem da uva Crimson em secador de bandeja por convecção forçada, com isso ajustar os modelos semiteóricos aos dados experimentais e obter o valor da difusividade efetiva (Def). As curvas de secagem foram obtidas utilizando-se um secador de bandeja por convecção forçada a $70^{\circ} \mathrm{C}$. Os dados experimentais foram ajustados empregando-se os modelos de Page, Newton, Henderson e Pabis, Wang e Singh, Midilli e Logarítmico, utilizando regressão não-linear. Todos os modelos apresentaram coeficiente de determinação $\left(R^{2}\right)$ superior a 0,98 . Dentre os modelos aplicados observou-se que o modelo de Wang e Singh foi o que apresentou melhor ajuste. A difusividade efetiva da uva Crimson foi calculada a partir do modelo difusional de Crank (1975) e apresentou um valor da difusividade de $2,18 \times 10^{-10} \mathrm{~m}^{2} \mathrm{~s}^{-1}$. Desta forma, pode-se concluir que para os frutos a secagem pode ser uma alternativa viável na produção de novos produtos, melhorando o desempenho econômico das indústrias alimentícias.
\end{abstract}

\section{INTRODUÇÃ̃O}

A variedade de uva Crimson foi obtida pelo programa de melhoramento genético do Serviço de Pesquisa Agrícola do Departamento de Agricultura dos Estados Unidos (USDA/ARS), lançada na Califórnia em 1989 e introduzida no Brasil pelo Instituto Agronômico de Campinas no mesmo ano (SOARES e LEÃO, 2009; POMMER et al., 1999). E esta e consumida na sua forma in natura ou passarem por um processo de secagem para a produção de uva passa (ALMEIDA, 2013)

A secagem é um processo em que a água é retirada para prevenir ou adiar o crescimento de microrganismos nocivos, assim como certas reações químicas. Além da conservação, a secagem, é utilizada para reduzir o custo ou dificuldade de embalagem, manipulação, armazenagem e transporte dos alimentos, com redução do peso e volume (BARBOSA-CÁNOVAS e VEGAMERCADO, 2000).

Estudos e análises de curvas de secagem e determinação do teor de água permitem entender e visualizar melhor o processo de secagem, bem como escolher o procedimento, o tratamento, o equipamento e a temperatura adequada para se realizar a desidratação de fruta, para melhor qualidade sensorial e tecnológica (OLIVEIRA et al., 2002).

A difusividade efetiva (Def) engloba os efeitos de todos os fenômenos que podem intervir sobre a migração da água e seu valor é sempre obtido pelo ajuste de valores experimentais. Pode-se entender a difusividade como a facilidade com que a 
água é removida do material. Como a difusividade varia conforme mudam as condições de secagem (temperatura e velocidade do ar), ela não é intrínseca ao material; convenciona-se, então, chamá-la de difusividade efetiva (OLIVEIRA et al., 2006).

Os objetivos deste trabalho foram obter a curva de secagem da uva Crimson em um secador convectivo, para obter os ajustes de modelos semiteóricos aos dados experimentais definindo o melhor modelo ajustado e calcular a difusividade efetiva (Def) da uva Crimson.

\section{MATERIAIS E MÉTODOS}

\subsection{Matéria Prima}

Os experimentos foram realizados no Laboratório de Tecnologias Alternativas da Universidade Federal de Sergipe, campus de São Cristóvão - SE.

A uva Crimson foi adquirida em comercio local. Os frutos foram selecionados de acordo com a qualidade, uniformidade e grau de maturação.

Os frutos selecionados foram lavados em água, sanitizados com solução de hipoclorito de sódio a 200ppm permanecendo durante 15 minutos nesta solução e posteriormente foram enxaguados com solução de hipoclorito de sódio a 5,0ppm para remoção do excesso de hipoclorito. Após a higienização as uvas foram colocadas nas bandejas para secagem em secador convectivo-PARDAL mod. PE 100. Para avaliar o processo de encolhimento das uvas antes e após a secagem, foram selecionados três frutos para medida de seus diâmetros com paquímetro.

A massa seca da uva Crimson foi determinada pelo método de secagem em estufa de esterilização e secagem- BIOPAR a $70{ }^{\circ} \mathrm{C}$.

\subsection{Secagem}

Para determinação da curva de secagem, foi utilizado um secador convectivo PARDAL mod. PE 100 á $70^{\circ} \mathrm{C}$. As bandejas foram pesadas em intervalos de 20 minutos nas primeiras três horas. Posteriormente, a pesagem foi realizada a cada 30 minutos, durante o restante do decorrer da secagem até atingir um peso constante.

Os dados de secagem foram ajustados com os modelos da Tabela 1. As equações matemáticas foram empregadas como correlações empíricas para descrever o comportamento de secagem. Estas equações expressam a razão de umidade (MR) em função do tempo.

Tabela 1. Os modelos matemáticos aplicados à curva de secagem da uva Crimson.

\begin{tabular}{cc}
\hline Nome do Modelo & Equação \\
\hline Newton & $M R=\exp (-k t)$ \\
Page & $M R=\exp \left(-k t^{n}\right)$ \\
Midilli & $M R=\exp \left(-k t^{n}\right)+b t$ \\
Logarítmico & $M R=a \exp (-k t)+b t$ \\
Wang e Singh & $M R=1+\mathrm{at}+\mathrm{bt}{ }^{2}$ \\
Henderson e Pabis & $M R=a \exp (-k t)$ \\
\hline
\end{tabular}

Fonte: DURIGON (2013)

Onde MR é dado por:

$M R=\frac{M-M e}{M 0-M e}$

Os ajustes dos modelos aos dados experimentais de secagem foram realizados mediante procedimento de regressão não linear do pacote Statistica (StatSoft, versão 7, Tulsa, EUA). Os critérios de escolha dos melhores ajustes foram baseados na determinação do coeficiente de regressão $\left(R^{2}\right)$, gerado pelo próprio software estatístico e o valor do qui-quadrado reduzido $\left(\chi^{2}\right)$, o valor de $\chi^{2}$ leva em consideração as respostas observadas experimentalmente e os valores preditos pelo modelo, como mostrado na Equação (2) 
$\chi^{2}=\frac{\sum_{i=1}^{N}(\text { MRexp }, i-M R p r e v, i)^{2}}{N-Z}$

Foi considerado que as uvas apresentavam geometria esférica, logo se pode utilizar a Equação 3, proposta por Crank (1975), a fim de obter o valor da difusidade efetiva (Def). Foi utilizado método de ajuste não linear do software Statistica (StatSoft, versão 7, Tulsa, EUA), no qual buscou-se simplificar a Equação 3 até o termo que apresentasse um coeficiente de regressão linear $\left(R^{2}\right)$ superior a 93\%, sendo este nível (valor de $n$ ) considerado para cálculo direto da difusividade efetiva. Para facilitar a aplicação da equação no software Statistica, substitui-se a porção constante do somatório por uma variável, tal qual a Equação 4.

$M R=\frac{6}{\pi^{2}} \sum_{n=1}^{\infty} \frac{1}{n^{2}} e^{\left[-n^{2} \frac{\pi^{2} D e f}{r^{2}} t\right]}$

$z=\frac{\pi^{2} D e f}{r^{2}}$

Portanto, a Equação 3 pôde ser transformada na Equação 5:

$M R=\frac{6}{\pi^{2}} \sum_{n=1}^{\infty} \frac{1}{n^{2}} e^{\left[-n^{2} z t\right]}$

\section{RESULTADOS E DISCUSSÃO}

O teor de umidade final para a uva Crimson foi de 13,01\% (b.u), a Figura 1 apresenta a curva de secagem na temperatura de $70{ }^{\circ} \mathrm{C}$ e os ajustes dos modelos presentes na Tabela 1 estão apresentados na Figura 2.

O elevado tempo necessário para a secagem desta fruta se deve ao fato desta conter uma membrana cuticular contínua cobrindo a epiderme. Esse suporte carrega um filme fino de cera, de natureza hidrofóbica, que estabelece uma barreira efetiva contra a perda de água por parte do fruto. Desta forma, faz-se necessário retirar essa cera ou modificar sua estrutura, tornando-a hidrofílica, com o auxílio de pré-tratamentos químicos, antes de submeter à uva ao processo de desidratação (GABAS, 1998).

Figura 1. Cinética de secagem para uva Crimson a $70{ }^{\circ} \mathrm{C}$.

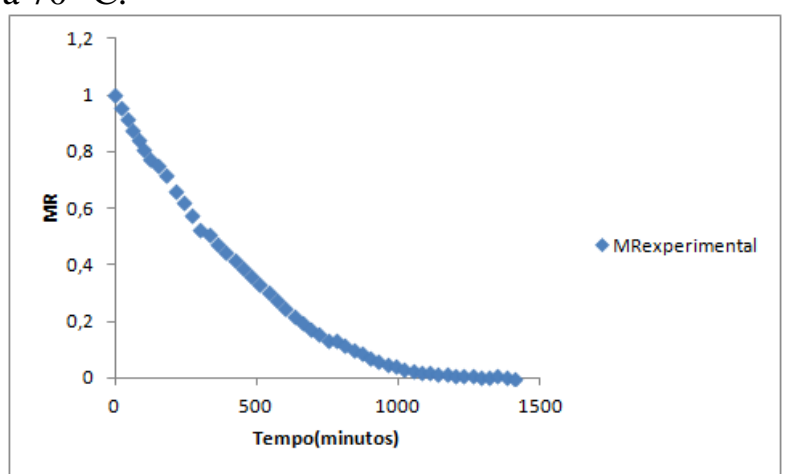

Fonte: AUTOR(2015)

Figura 2. Cinética de secagem com os ajustes da curva de secagem pelos modelos: (a) modelo de Newton, (b) modelo de Page, (c) modelo de Midilli, (d) modelo logarítmico, (e) modelode Wang e Singh e (f) modelo de Henderson e Pabis para uva Crimson a $70^{\circ} \mathrm{C}$.

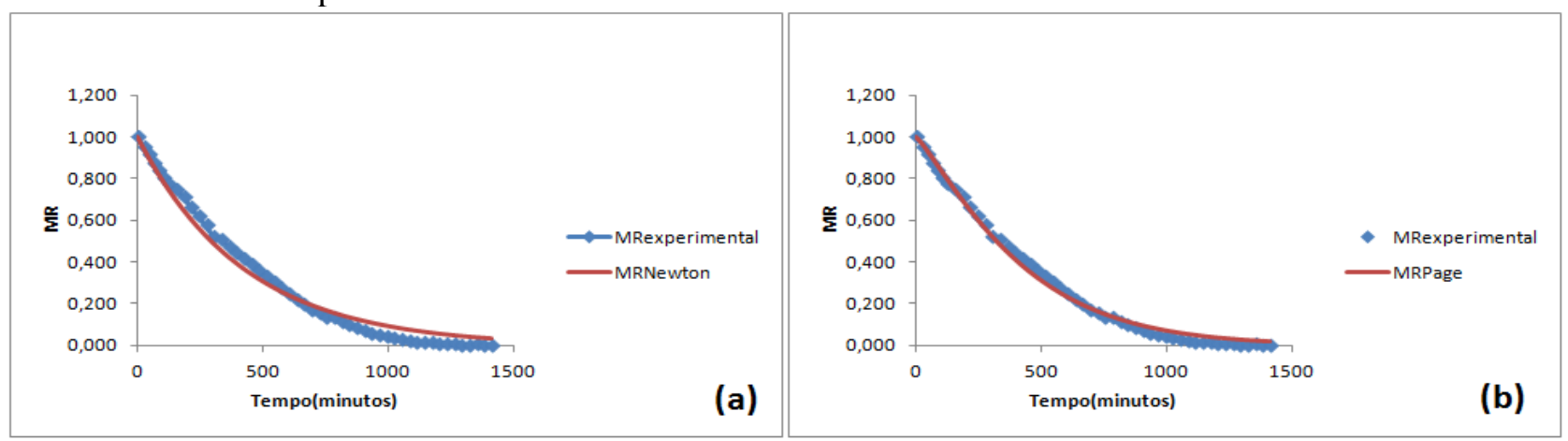




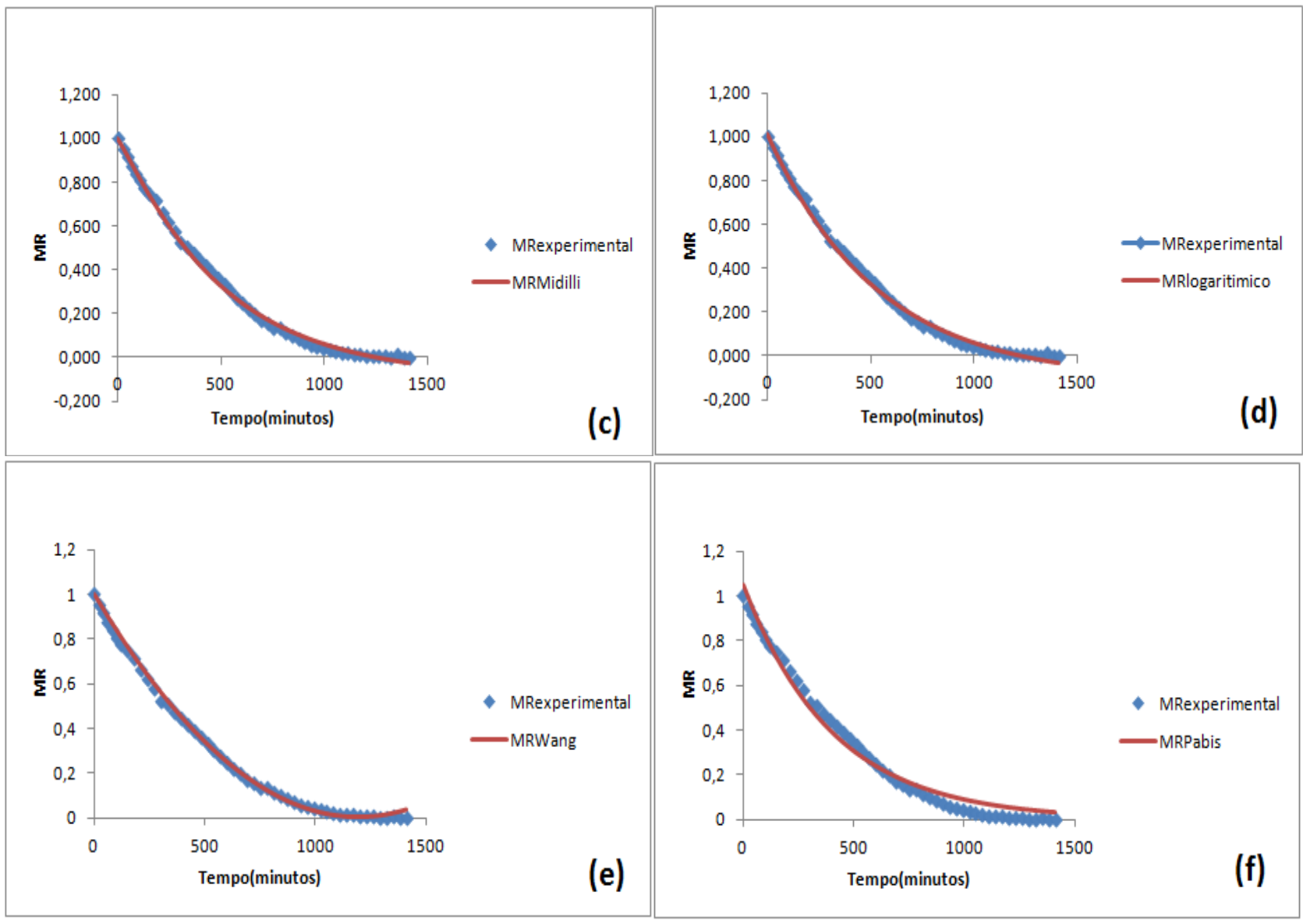

Fonte: AUTOR (2015)

A partir da Figura 1, pode-se verificar que na temperatura estudada, a razão de umidade reduziu rapidamente no início e posteriormente diminuiu lentamente à medida que aumentava o tempo de secagem. Esses resultados estão de acordo com a anterior observação de diferentes secagens de alimentos (KARABULUT et al., 2007; LAHSASNI et al., 2004).

Na Tabela 2 estão os parâmetros estatísticos utilizados para a comparação entre os seis modelos de secagem analisados, na condição de secagem estudada para esta fruta e o valor das constantes dos modelos.

Para a temperatura utilizada na secagem da uva, verifica-se que todos os modelos matemáticos ajustados aos dados experimentais apresentaram coeficientes de determinação $\left(R^{2}\right)$ superiores a 0,98 , sendo o modelo de Wang et al., (2007) o que apresentou maior valor de $R^{2}$ na temperatura de secagem estudada. Devido ao fato de que o coeficiente de regressão $\left(R^{2}\right)$ sozinho não constituir um bom critério para a seleção de modelos não lineares (MADAMBA et al., 1996), faz-se necessário a verificação de outro parâmetro, como o qui-quadrado reduzido $\chi^{2}$. Foi verificado que o modelo Wang et al., (2007) obteve o menor valor de $\chi^{2}$, uma vez que quanto maior o $R^{2}$ e menor os valores $\chi^{2}$, melhor será o procedimento de ajuste (TOGRUL; PEHLIVAN, 2004; MAZUTTI et al, 2010;. SANJINEZ-ARGANDOÑA et al., 2011). 
Tabela 2. Os modelos matemáticos aplicados às curvas de secagem da uva Crimson, com os valores das constantes dos modelos.

\begin{tabular}{ccccccc}
\hline Nome do Modelo & $\mathbf{k}$ & $\mathbf{n}$ & $\mathbf{a}$ & $\mathbf{b}$ & $\mathbf{R}^{2}$ & $\boldsymbol{\chi}^{\mathbf{2}}$ \\
\hline Newton & 0,002352 & - & - & - & 0,99183 & $1,60 \times 10^{-3}$ \\
Page & 0,000755 & 1,182198 & - & - & 0,99738 & $5,22 \times 10^{-4}$ \\
Midilli & 0,001186 & 1,092512 & - & $4,6 \times 10^{-5}$ & 0,99871 & $2,56 \times 10^{-4}$ \\
Logaritímico & 0,001878 & - & 1,12287 & 0,11546 & 0,99843 & $3,13 \times 10^{-4}$ \\
Wang e Singh & - & - & 0,001671 & $10^{-6}$ & 0,99889 & $2,22 \times 10^{-4}$ \\
Herdenson e Pabis & 0,002466 & - & 1,04951 & - & 0,99318 & $1,36 \times 10^{-4}$ \\
\hline
\end{tabular}

Fonte: Autor(2015)

O diâmetro médio do encolhimento das uvas foi de 7,02 mm, o que leva a um valor de raio esférico de $3,51 \mathrm{~mm}$, ou $0,00351 \mathrm{~m}$. Os dados apresentaram desvio padrão de 0,078 $\mathrm{mm}$.

Durante a análise por ajuste não linear, verificou-se que no nível $\mathrm{n}=8$ os valores de $R^{2}$ foram superiores a 93\%, sendo então considerado tal nível da equação de Crank (1975) (Equação 6):

$M R=\frac{6}{\pi^{2}}\left(e^{(-z t)}+\frac{1}{4} e^{(-4 z t)}+\frac{1}{9} e^{(-9 z t)}+\right.$ $\frac{1}{16} e^{(-16 z t)}+\frac{1}{25} e^{(-25 z t)}+\frac{1}{36} e^{(-36 z t)}+$

$\left.\frac{1}{49} e^{(-49 z t)}+\frac{1}{64} e^{(-64 z t)}\right)$

Realizando o ajuste desta equação aos valores de adimensional de umidade (MR) e tempo de secagem (t) do experimento, obtevese o valor de $\mathrm{z}$ para e consequentemente, $\mathrm{o}$ valor da Def, segundo a Equação 4.

Pôde-se verificar que a difusividade efetiva no experimento foi de $2,18 \times$ $10^{-10} \mathrm{~m}^{2} \mathrm{~s}^{-1}$, valores de acordo com a constatação de Zogzas, Mauroulis e MarinosKouris (1996), que postulou que para produtos alimentícios, os valores de Def se encontram na faixa de $10^{-11}$ a $10^{-9} \mathrm{~m}^{2} \mathrm{~s}^{-1}$.

\section{CONCLUSÃO}

O comportamento da curva de secagem da uva Crimson é o mesmo que os obtidos para a maioria dos produtos agrícolas e o tempo de secagem foi de $24 \mathrm{~h}$ para a temperatura de secagem a $70^{\circ} \mathrm{C}$.

$\mathrm{O}$ método de secagem não foi muito eficiente, visto que foi necessário um longo tempo de secagem. Mas este pode ser adotado no caso da produção de orgânicos, onde não é permitida à adição de substancias química no processamento.

O modelo matemático de secagem proposto por Wang et al., (2007) foi que se ajustou melhor com relação aos dados experimentais. A difusividade efetiva (Def) da uva Crimson foi de $2,18 \times 10^{-10} \mathrm{~m}^{2} \mathrm{~s}^{-1}$.

\section{NOMENCLATURA}

MR Razão de umidade, (adimensional);

$k$ Constante de secagem, $\left(\mathrm{min}^{-1}\right)$;

$t$ Tempo, (min);

$n, \quad a, b$ Constantes experimentais, (adimensionais);

$e$ Expoente;

M Massa no tempo t, (g);

Me Massa de equilíbrio, (g);

M0 Massa inicial, (g);

$\mathrm{R}^{2}$ Coeficiente de regressão linear, (adimensional)

$\chi^{2}$ Qui-quadrado reduzido (adimensional)

MRexp, i e MRpred, i Razão de umidade experimental e a prevista respectivamente, (adimensional);

N É o número de observações, (adimensional);

Z É o número de parâmetros, (adimensional);

Def Difusividade efetiva, $\left(\mathrm{m}^{2} \mathrm{~s}^{-1}\right)$; 
z Constante da equação de Crank, $\left(\min ^{-1}\right)$

$n$ É o nível do somatório da equação de Crank, (admensional);

$\mathrm{r}$ Valor médio do raio do encolhimento da uva Crimson, (m).

\section{REFERÊNCIAS}

ALMEIDA L.C.P.Desidratação Osmótica e Secagem Convectiva de Uvas da Cultivar Crimson. 23 p., Dissertação (mestrado) Universidade Federal de Santa Catarina, Centro Tecnológico. Programa de Pós-Graduação em Engenharia de Alimentos. Florianópolis, SC, 2013.

BARBOSA-CÁNOVAS, G. V.; VEJAMERCADO, H. Deshidratación de alimentos. Zaragoza: Editorial AcribiaS. A., 2000.

CRANK, J. The mathematics of diffusion. 2. ed. Oxford: S.I. Claredon Press, 1975. $414 p$.

KARABULUT, I.; HAYALOGLU, A. A.; YILDIRIM, H. Thin-layer drying characteristics of kurut, a Turkish drieddairy by-product. International Journal of Food Science and Technology, v. 42, n. 9, p. 10801086, 2007.

LAHSASNI, S.; $\quad$ KOUHILA, M.; MAHROUZ, M.; JAOUHARI, J. T. Drying kinetics of prickly pear fruit (Opuntia ficus indica). Journal of Food Engineering, v. 61, n. 2, p. 173-179, 2004.

MADAMBA, P. S.; DRISCOLL, R. H.; BUCKLE, K. A. Thin-layer drying characteristcs of garlic slices. Journal of Food Engineering, Essex, v. 29, n. 1, p. 7597, 1996.

MAZUTTI, M. A.; ZABOT, G.; BONI, G.; SKOVRONSKI, A.; OLIVEIRA, D.; DI LUCCIO, M.; OLIVEIRA, J. V.; RODRIGUES, M. I.; TREICHEL, H.;
MAUGERI, F. Mathematical modeling of thin-layer drying of fermented and nonfermented sugarcane bagasse. Biomass and Bioenergy, v. 34, n. 5, p. 780-786, 2010.

POMMER, C. V.; TERRA, M. M.; PIRES, E. J. P.; PASSOS, I. R. S.; MARTINS, F. P. Introdução dos cultivares de uva de mesa 'Fantasia' e 'Ruiva' no Brasil. Sci. agric. vol. 56, n.1, p. 247 - 252, Piracicaba - SP. 1999.

OLIVEIRA, L. F.; NASCIMENTO, M. R. F.; BORGES, S. V.; RIBEIRO, P. C. N.; RUBACK, V. R. Aproveitamento alternativo da casca do maracujá-amarelo (Passiflora edulis F. flavicarpa) para produção de doce em calda. Ciência e Tecnologia de Alimentos, v. 22, n. 3, p. 254-258, 2002.

OLIVEIRA, R. A.; Oliveira, W. P.; Park, K. J. Determinação da difusividade efetiva de raiz de chicória. Engenharia Agrícola, v.26, p.181-189, 2006.

SANJINEZ-ARGANDONA, E. J.; BRANCO, I. G.; BITTENCOURT, T. U.; MUNHOZ, C; L; Influência da geometria e da temperatura na cinética de secagem de tomate (Lycopersicum esculentum). Ciência e Tecnologia deAlimentos, v. 31, n. 2, p. 308312, 2011.

SOARES, J. M; LEÃO, P. C. S; Editores técnicos, A viticultura no Semiárido brasileiro. Brasília, DF: Embrapa Informações Tecnológica; Petrolina: Embrapa Semi-Árido, 756 p., 2009.

WANG, Z.; SUN, J.; LIAO, X.; CHEN, F.; ZHAO, G.; WU, J.; HU, X. Mathematical modeling on hot air drying of thin layer apple pomace. Food Research International, Toronto, v. 40, p. 39-46, 2007. 
ZOGZAS, N. P.; MAUROULIS, Z. B.;

MARINOS-KOURIS, D. Moisture diffusivity data compilation in foodstuffs. Drying Technology, New York, v.14, n.10, p. 22252253, 1996. 